\title{
Design of Adaptive Filter for Biosignal Noise Cancellation Using Brent Kung Adder
}

\author{
Gomathi.S ${ }^{1}$, Dr.S.Sasikala ${ }^{2}$, Meenakshi K ${ }^{3}$,D.Karpagapriya ${ }^{4}$, S.Ilakiya, \\ ${ }^{1,3}$ Assistant Professor, Department of ECE, Kongu Engineering College, India, samgomathi@ gmail.com \\ ${ }^{2}$ Associate Professor, Department of ECE, Kongu Engineering College, India, sasikalapriyaadarsan@gmail.com \\ ${ }^{3,4,5}$ Student, Department of ECE, Kongu Engineering College, India
}

\begin{abstract}
Adaptive filter can update itself with variation in time. Thus it finds its application in variety of signal processing tasks such as System identification, prediction, Inverse Modeling and noise cancellation. When the priori of the signal is unknown, adaptive filters play an important role as they are self-regulating system that uses the recursive algorithm for processing. This paper demonstrates the design of Kalman adaptive filter using parallel prefix adder, the Brent Kung Adder for noise cancellation in ECG signal. Experiments are carried out with traditional adder and the Brent Kung Adder in Kalman Adaptive Filter. The Performance of the filter is analysed in terms of Signal to Noise Ratio and is compared for 4,8,16,32 tap filters and the proposed adder implementation improves the performance of the filter.
\end{abstract}

Key words :Adaptive Filter, ECG Signal, Brent Kung Adder Adder, Signal to Noise Ratio

\section{INTRODUCTION}

According to an optimization algorithm, Adaptive filter is a system with linear filter that has a transfer function controlled by variable parameters and means to adjust those parameters. Almost all adaptive filters are digital filters due to the complexity of the optimization algorithms. In practical situations, the system is operating in an uncertain environment where the input condition is not clear and/or the unexpected noise exists. A highly successful solution to this more difficult is found in the "adaptive filter", which is a powerful system with a wide of engineering applications. [9-13].Adaptive filters consist of three basic components: the adaptive filter $h(n)$, the error $E(n)$ and the adaptation function $: Y(n)=X(n) * H(n)$ and as shown in Figure 1.

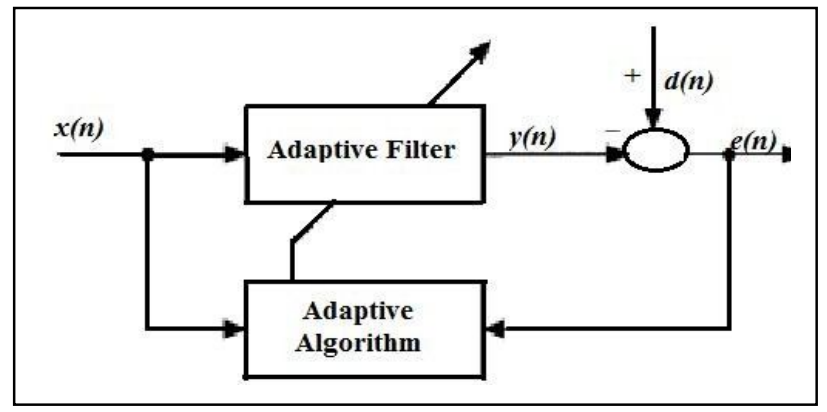

Figure 1: Adaptive Filter-Block Diagram
The goal of the system is to adapt the filter in such a way that the input signal $\mathrm{X}(\mathrm{n})$, is filtered an output signal $\mathrm{Y}(\mathrm{n})$, that will minimize the error signal $\mathrm{E}(\mathrm{n})$, when subtracted from the specified signal $D(n)$. The arrow through the adaptive filter is standard notation to point that the filter is adaptive. This means that all of the filter coefficients can be adjusted in such a way that the mean square error is to be minimized.[16][17] To ensure the steadiness of the adaptive algorithm, most adaptive filters use an FIR type. The adaptive filters are widely utilized in areas like control systems, communications, signal processing, acoustics, to affect random signals with stationary or quasi stationary statistics.

A variety of research papers are available based on adaptive filter and parallel prefix adder. Implementation of PPA-Brent Kung Adder For Computing Application[1][2][5]. They implemented parallel prefix adder and it provided high speed and reduced delay arithmetic operations. There are few literatures related to design of efficient Han Carlson Adder[3]. In this method has wider word length and the parallel prefix adder is replaced as speculative Han-Carlson adder[6] is introduced as variety stages of Brent-Kung and Kogge-Stone adders reduces the complexity of adder design. Parallel prefix adders are used to speed up the binary additions as they are very flexible.[4][5].High level transformation for adaptive filter design has been discussed for area optimisation[9][12][14-15][16,19].High Speed adders and Multipliers has been implemented for various applications.[18]

\section{KALMAN ADAPTIVE ALGORITHM}

KALMAN algorithm is an algorithm that uses a series of measurements observed over time, containing statistical noise and other inaccuracies, and produces estimates of unknown variables that tend to be more accurate than those based on a single measurement alone, by estimating a joint probability distribution over the variables for each timeframe. The KALMAN filter finds applications in various technologies such as navigation and control of vehicles, motor system etc.,. These filters are mostly used in the field of robotic motion planning and control and also included in trajectory optimization. Evaluation of the current state variables along with their uncertainties is done by KALMAN filter. It does not need any additional past information. When the outcome of the next measurement is observed the estimates are updated. 
Step 1: Compute KALMAN gain

$g(t+1)=k(t) u(t+1) /(u H(t+1) k(t) u(t+1)+Q M)$

Step 2: Filtered output

$y(t+1)=u H(t+1) w(t+1)$

Step 3: Error estimation

$e(t+1)=d(t+1)-y(t+1)$

Step 4: Update the coefficient

$w(t+1)=w(t)+e(t+1) g(t+1)$

Step 5: Compute correlation matrix

$k(t+1)=k(t)-g(t+1) u H(t+1) k(t)+Q P$

where $\mathrm{QM}=\delta-1 \mathrm{IM}$ and $\mathrm{QP}=\delta-1 \lambda$

Complexity of KALMAN algorithm is low compared to RLS and AFFINE since matrix operations are not required.

\section{BRENT KUNG ADDER}

Adders are an integral part of any system that find applications in Arithmetic and Logic Units (ALUs), microprocessors and memory addressing units. The need for a Parallel Prefix Adder (PPA) is that the computation time is faster than the other adders. Let $\mathrm{A}=\mathrm{an}-1 \ldots \mathrm{a} 1 \mathrm{a} 0$ and $\mathrm{B}=$ bn-1 ... b1 b0 be the n-bit augend and n-bit addend respectively, then binary addition is defined by the equations (6) and (7)

$S i=a i \oplus b i \oplus c i(6)$

$c i=a i b i+a i c i+b i c i(7)$

Following are the three steps used design of the structure of Brent Kung adder.

\subsection{Pre-processing}

Pre-processing, involves creation of generate and propagate signals. Pre-processing, involves creation of generate and propagate signals. Generate $\left(\mathrm{g}_{i}\right)$ and propagate $\left(\mathrm{p}_{\mathrm{i}}\right)$ signals are defined by the equations (8) and (9) respectively based on prefix computation.

$g i=a i \cdot b i$

$p i=a i \oplus b i$

\subsection{Prefix Computation}

PPA construction depends on the notion of group carry propagate and group generate signals. Group generate and group propagate signals are defined by the equations (10) and (11) respectively.

$G[i: j]=G[i: j]+P[i: j] \cdot G[j-1: k](10)$

$P[i: j]=P[i: j] \cdot P[j-1: k](11)$

Group generate and group propagate signals are generated to as defined by the equation (12) as

$(G, P)[i: k]=(G, P)[i: j] *(G, P)[j-1: k](12)$

\subsection{Post-processing}

Formation of carry and sum bits for each individual operand bit is involved in Post-processing step. The equations for carry and sum are defined as per equations (13) and (14) respectively.

$C i=G[i: 0](13)$

$S i=p i \oplus c i-1(14)$

\section{4 Structure of Brent Kung adder}

Figure 2 shows the working of Four bit Brent Kung adder. The inputs are $\mathrm{A}$ and $\mathrm{B}$ and carry $\mathrm{C}_{\mathrm{in}}$. The outputs are calculated using the three stages. Let the input be $\mathrm{A}=0011$, B $=0101$ and $\mathrm{C}_{\mathrm{in}}=1$ and the result obtained in simulation is shown in Figure 3

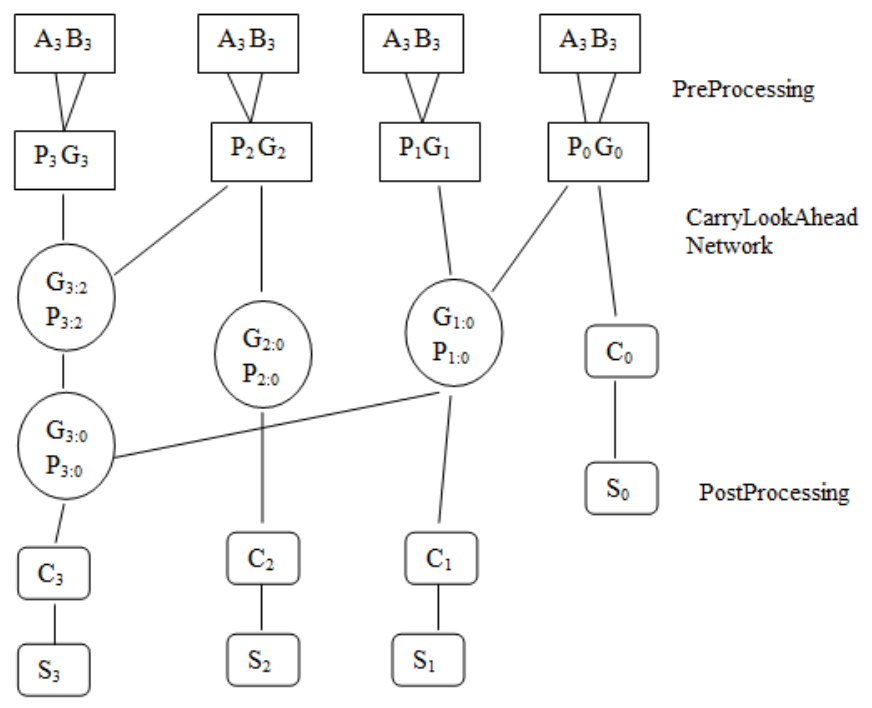

Figure 2: Brent Kung Adder

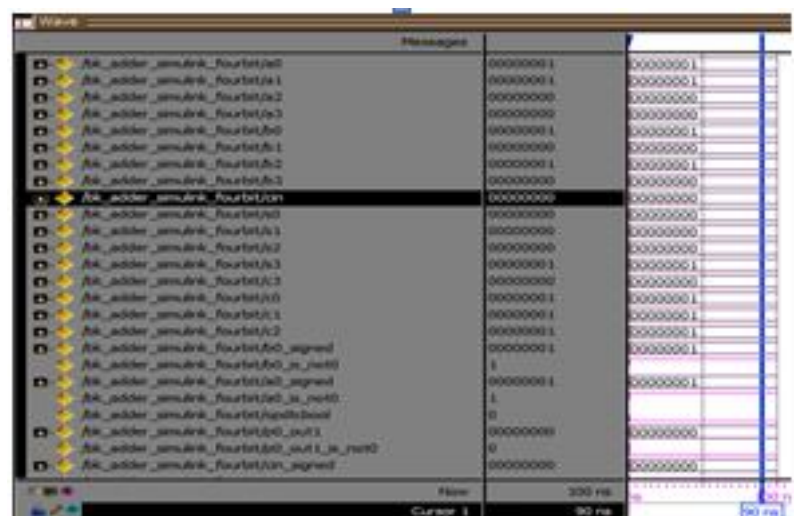

Figure 3: Output ofBrent Kung Adder

\section{FILTER DESIGN USING BRENT KUNG ADDER}

Figure 4 shows the Eight Tap Conventional KALMAN Adaptive Filter structure is designed and simulated in Simulink. 


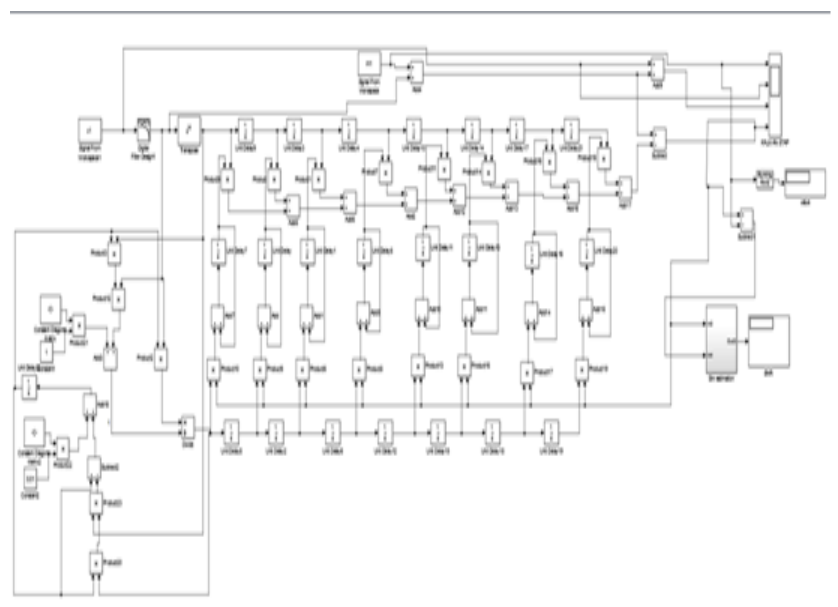

Figure 5 shows the eight tap KALMAN adaptive filter designed using Four bit Brent kung adder in FIR and Weight updation part is designed and simulated in Simulink.

Figure 4: Conventional Kalman Filter of order 8

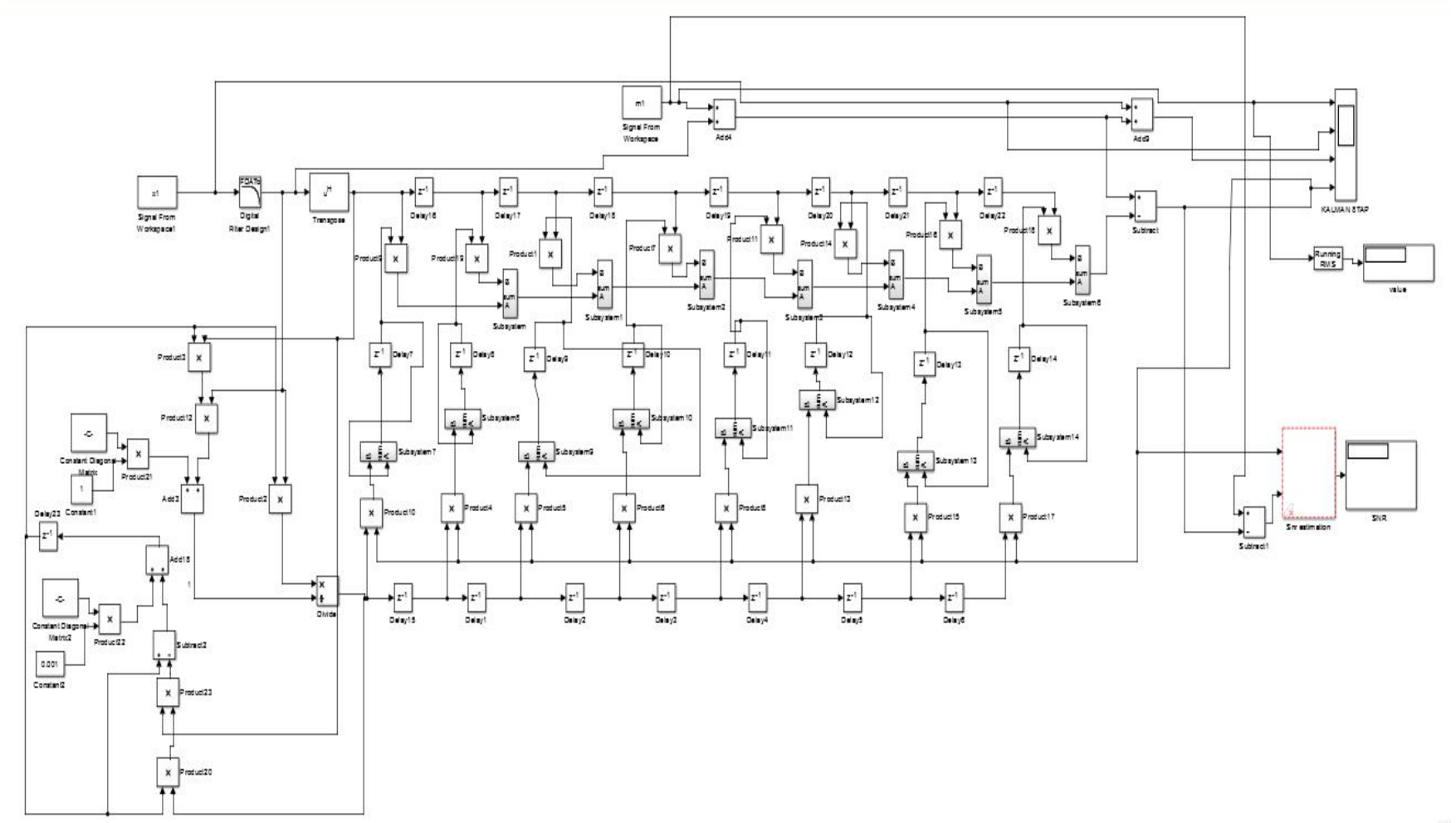

Figure 5:8-Tap KALMAN Adaptive Filter Designed using Four bit Brent Kung Adder in FIR and Weight UpdationPart

\section{SIMULATION RESULTS}

The Kalman adaptive filter is designed using conventional type, Brent Kung adder in FIR part and Brent kung adder both in FIR and Weight Update part. The ECG signal obtained from MIT BIH database is taken as the input for the performance evaluation of Kalman filter. Kalman filters with filter order 4,8,16 are designed and simulated. The ECG signal used for the analysis is contaminated with Power
Line Interference (PLI) noise. ECG Signal contaminated with PLI noise of $50-60 \mathrm{~Hz}$ is applied as input signal to the designed filters. The simulations result for 8 tap Conventional Kalman Adaptive Filter is shown in Figure 6. 


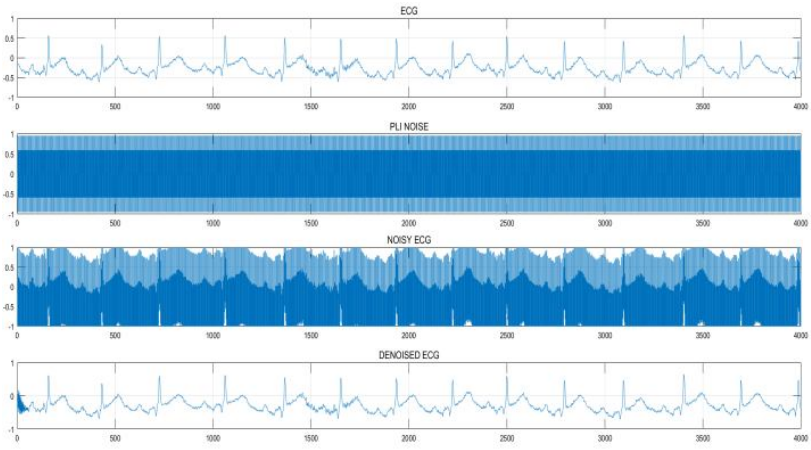

Figure 6: Simulation Output ofConventionalKalman Filter of order 8 using

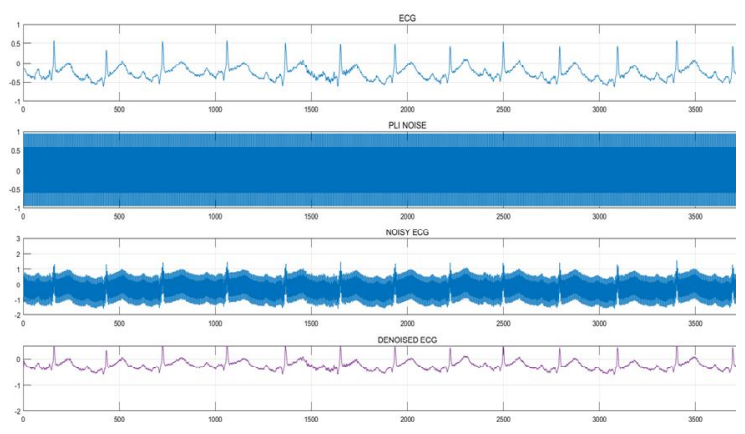

Figure 7: Simulation Output ofKalman Filter of order 8 using Four-bit Brent Kung adder in FIR part and Weight Updation part

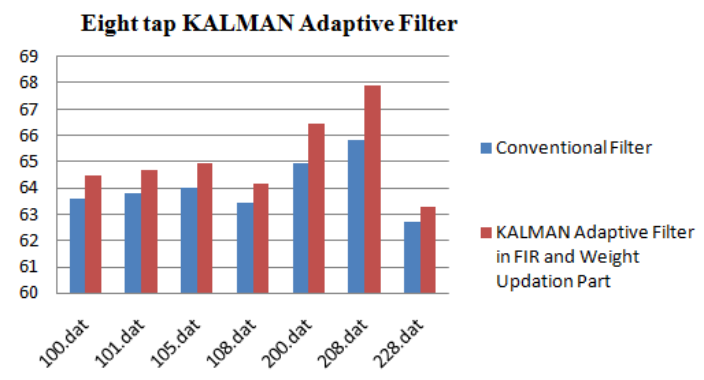

Figure 8: SNR comparison of Conventional and Proposed Filters

Figure 7 shows the simulation output of Eight Tap Kalman Adaptive Filter with modified adder in FIR part and weight updation part in Simulink. Figure 8 reveals the Signal to Noise Ratio for the conventional and proposed filters. It is observed that performance of the filter is improved.

\section{CONCLUSION}

The Brent Kung adder logic is applied to improve the performance of the adaptive filtering algorithms. This method is used to reduce the number of functional units in the FIR part and weight updation part. The Kalman structure is designed and simulated in Simulink with both conventional and proposed designs. KALMAN Adaptive filter using Brent Kung adder both in FIR part and weight updation part shows an SNR improvement of $26.7 \%$ and $32 \%$ than Conventional filter design for various ECG signals. It was also inferred that when the length of the filter was increased, there was a significant improvement in signal to noise ratio and minimal reduction in the mean square error. Hence usage of parallel prefix adder like Brent Kung adder in KALMAN Adaptive filter has a performance improvement than Conventional filter design.

\section{REFERENCES}

1.Shivani Parmar and Kirat Pal Singh,Design of High Speed Hybrid Carry Select Adder, Proc of IEEE's 3rd International Advance Computing Conference (IACC) Ghaziabad, ISBN: 978-1-4673-4527-9,22-23 February 2018. 2. Yajaun He, Chip-Hong Chang, and Jiangmin Gu, An area efficient 64- Bit square Root carry-select adder for low power Applications, in Proc. IEEE International Symposium Circuits and Systems, vol. 4, pp. 4082- 4085,2018.

3. Y. Choi, Parallel Prefix Adder Design, in Proc. 17th IEEE Symposium on Computer Arithmetic, pp. 90-98, 27th June 2017.

4.A. K. Verma, P. Brisk, and P. Ienne, Variable Latency Speculative Addition: A New Paradigm for Arithmetic Circuit Design, in Proc.Design, Auto. Test Eur. (DATE'08), Mar. 2018, pp. 1250-1255.

5. P. M. Kogge and H. S. Stone, A parallel algorithm for the efficient solution of a general class of recurrence equations, IEEE Trans. Computer., vol. C-22, no. 8, pp. 786-793, Aug. 2017.

https://doi.org/10.1109/TC.1973.5009159

6. T. Han and D. Carlson, .Fast area-efficient VLSI Adders, In Proc. 8th Symp. Comp. Arithmetic, Sept. 2017, pp. 49.56.

7. S. Mukherjee, J. Emer, and S. Reinhardt, The soft error problem: an architectural perspective, High-Performance Computer Architecture, 2015. HPCA-11.11th International Symposium on, pp. 243-247, Feb.2015.

8. A. Johnston, G. Swift, and D. Shaw, Impact of cmos scaling on singleevent hard errors in space systems, Low Power Electronics, 2015., IEEE Symposium on, pp. 88-89, Oct 2015.

9. Gomathi Swminathan,G Murugesan,S Sasikala,L Murali, A novel implementation of combined systolic and folded architectures for adaptive filters in FPGA,Microprocessors and Microsystems, vol.74,2020.

https://doi.org/10.1016/j.micpro.2020.103018

10.Gomathi Swaminathan, Murugesan Govindaswamy and Sasikala Subramaniyam, Performance Analysis of Karatsuba Vedic Multiplier and Computation Sharing Multiplier in the Adaptive Filter Design, International Journal of Innovative Technology and Exploring Engineering, volume 9, no.2, pp. 4425-4429, Dec 2019

https://doi.org/10.35940/ijitee.B7454.129219

11.Gomathi, S,Murugesan, G \&Jayapravintha, Design Of Systolic Architecture For Various Adaptive Filters For Noise Cancellation, in Proc.of 3rd International Conference on Signal Processing, Communication and Networking (ICSCN),2015,IEEE Explorer

DOI: 10.1109/ICSCN.2015.7219907. 
12.Gomathi, S,Murugesan, G, Sasikala, S \&Chitra, M,Area Efficient Implementation of Adaptive filters using High World $\quad(I 2 C 2 S W)$, 2018, IEEE Explorer, 10.1109/I2C2SW45816.2018.8997199

13. S. Sasikala, G. Murugesan. Efficient digit serial architecture for sign least mean square adaptive filter for denoising of artifacts in ECG signals. International Journal of Biomedical Engineering and Technology 23 (2/3/4) (2017) 335-344

https://doi.org/10.1504/IJBET.2017.082672

14. Sasikala S, Gomathi S, Naveen kumar D, Praveenraaj R K, Priyadharshini B. FPGA Implementation of Adaptive LMS Lattice Filter. Bioscience Bitechnology Research Communications, Special Issue, Volume 13, No.3, pp. 121-125(2020).

15. C. Sathya, S. Sasikala, G. Murugesan, Denoising ECG signal using combination of ENSLMS and ZALMS algorithms, Proceedings of 3rd International Conference on Signal Processing, Communication and Networking, August 2015, DOI: 10.1109/ICSCN.2015.7219911 .

16. M.Chitra, S.Sasikala, S.Gomathi, A.Neetheswaran, C.Reetha. Design of Cascaded Adaptive Filter for ECG Denoising Applications. International Journal of Emerging Trends in Engineering Research,Vol. 8, No. 5, May 2020. https://doi.org/10.30534/ijeter/2020/61852020

17.S. Mula, V. C. Gogineni, A. S. Dhar, Algorithm and VLSI architecture design of proportionate-type LMS adaptive filters for sparse system identification,IEEE Trans. Very Large Scale Integr. (VLSI) Syst.,vol. 26, no. 9, pp. 1750-1762, Sep. 2018.

https://doi.org/10.1109/TVLSI.2018.2828165

18.Dr.S.Sasikala, S.Gomathi, M.Kanimozhi,, K.S.lakshanan, R.Karthik,Performance Analysis of a Low Power High Speed Hybrid Multiplier Circuit,International Journal of Advanced Trends in Computer Science Engineering,Vol.No.9,No.3,May 2020.

https://doi.org/10.30534/ijatcse/2020/197932020

19.Gomathi.S, Dr.S.Sasikala, D.Karpagapriya, S.Kowsalya, M.S.Karan Kishore Ananth, Design of Low Area and High Speed Parallel FIR Digital Filter using Fast FIR
Level Transformation, in Proc of International Conference on Intelligent Computing and Communication for Smart Algorithm, International Journal of Emerging Trends in Engineering Research, Vol. 8, No. 6, June 2020. https://doi.org/10.30534/ijeter/2020/100862020 